\title{
A novel point-of-care testing strategy for sexually transmitted infections among pregnant women in high-burden settings: results of a feasibility study in Papua New Guinea
}

Steven G. Badman ${ }^{*}$, Lisa M. Vallely ${ }^{1}$, Pamela Toliman², Grace Kariwiga ${ }^{3}$, Bomesina Lote ${ }^{4}$, William Pomat ${ }^{2}$, Caroline Holmer ${ }^{5}$, Rebecca Guy ${ }^{1}$, Stanley Luchters ${ }^{6,7}$, Chris Morgan ${ }^{6}$, Suzanne M. Garland ${ }^{8,9}$, Sepehr Tabrizi ${ }^{8}$, David Whiley ${ }^{10}$, Stephen J. Rogerson ${ }^{11}$, Glen Mola ${ }^{12}$, Handan Wand ${ }^{1}$, Basil Donovan ${ }^{1,13}$, Louise Causer ${ }^{1}$, John Kaldor ${ }^{1}$ and Andrew Vallely ${ }^{1}$

\begin{abstract}
Background: Sexually transmitted and genital infections in pregnancy are associated with an increased risk of adverse maternal and neonatal health outcomes. High prevalences of sexually transmitted infections have been identified among antenatal attenders in Papua New Guinea. Papua New Guinea has amongst the highest neonatal mortality rates worldwide, with preterm birth and low birth weight major contributors to neonatal mortality. The overall aim of our study was to determine if a novel point-of-care testing and treatment strategy for the sexually transmitted and genital infections Chlamydia trachomatis (CT), Neisseria gonorrhoeae (NG), Trichomonas vaginalis (TV) and Bacterial vaginosis (BV) in pregnancy is feasible in the high-burden, low-income setting of Papua New Guinea.
\end{abstract}

Methods: Women attending their first antenatal clinic visit were invited to participate. $C T / N G$ and TV were tested using the GeneXpert platform (Cepheid, USA), and BV tested using BVBlue (Gryphus Diagnostics, USA). Participants received same-day test results and antibiotic treatment as indicated. Routine antenatal care including HIV and syphilis screening were provided.

Results: Point-of-care testing was provided to 125/222 (56 \%) of women attending routine antenatal care during the three-month study period. Among the 125 women enrolled, the prevalence of CT was $20.0 \%$; NG, $11.2 \%$; TV, $37.6 \%$; and $B V, 17.6 \%$. Over half $(67 / 125,53.6 \%)$ of women had one or more of these infections. Most women were asymptomatic (71.6\%; 47/67). Women aged 24 years and under were more likely to have one or more STI compared with older women (odds ratio 2.38; $95 \%$ Cl: 1.09, 5.21). Most women with an STI received treatment on the same day (83.6\%; 56/67). HIV prevalence was $1.6 \%$ and active syphilis $4.0 \%$.

Conclusion: Point-of-care STI testing and treatment using a combination of novel, newly-available assays was feasible during routine antenatal care in this setting. This strategy has not previously been evaluated in any setting and offers the potential to transform STI management in pregnancy and to prevent their associated adverse health outcomes.

Keywords: Antenatal, Pregnancy outcome, Molecular testing, Syndromic management, Low-middle-income country, Genexpert, Point-of-care, STls

\footnotetext{
* Correspondence: sbadman@kirby.unsw.edu.au

${ }^{1}$ The Kirby Institute, University of New South Wales, Sydney, Australia

Full list of author information is available at the end of the article
} 


\section{Background}

Previous studies among antenatal women in lowresource settings have found high prevalences of sexually transmitted and genital infections, particularly Chlamydia trachomatis (CT), Neisseria gonorrhoeae (NG), Trichomonas vaginalis (TV) and Bacterial vaginosis (BV), that are readily curable with cheap antibiotics [1]. Untreated, these bacteria are associated with an increased risk of preterm birth, low birth weight, premature rupture of membranes and other adverse maternal and neonatal health outcomes [1].

Papua New Guinea (PNG) has among the highest neonatal mortality rates worldwide, with an estimated 23 neonatal deaths per 1,000 livebirths [2]. Preterm birth and low birth weight are major contributors to neonatal mortality; in PNG an estimated $16 \%$ of all infants are of low birth weight [3]. In addition, an estimated 15 infants per 1,000 births are stillborn [4]. Preliminary results from the country's first biobehavioural survey of STIs in pregnancy $(n=765)$ identified high prevalences of CT (23.1\%), NG (13.8\%), and TV (24.1\%) [5]. Overall, $44 \%$ of pregnant women had at least one of these infections [5]. Around $80 \%$ of women with laboratory-confirmed CT, NG or TV were asymptomatic and therefore would not have been detected and treated using national STI syndromic management guidelines that are based on clinical criteria alone, consistent with WHO recommendations for routine care in low-income settings [6, 7].

The lack of affordable, timely, easy-to-use and accurate STI tests that can be used at point-of-care has meant that for the last two decades routine practice in many low and middle-income countries (LMIC) has been based on syndromic management. Recent advances in STI diagnostics however may herald a new era in STI diagnosis and management in such settings, although this as yet is untested in a LMIC setting such as PNG.

The GeneXpert platform (Cepheid, Sunnyvale CA, USA) provides fully automated, easy-to-use point-ofcare molecular tests for CT, NG and TV that are as accurate as laboratory-based nucleic acid amplification tests (NAATs) [8]. This robust and portable platform has already revolutionized the diagnosis and management of tuberculosis in a number of LMIC [9] and is now being used in PNG. Disposable single-use cartridges hold the reagents for the simultaneous detection of CT and NG, with a separate cartridge used for TV detection. Test results are available in 90 (CT/NG) and 60 (TV) minutes, allowing for immediate management.

The platform requires specific training in test procedures, some infrastructure including reliable electrical power or a backup generator, as well as modifications to service provision and patient flow to allow for immediate treatment and contact tracing.
The diagnosis of BV has until recently relied on skilled microscopic examination and scoring of Gram stained vaginal smears using Nugent or Amsel scores. The BVBlue test (OSOM BVBlue Test, Gryphus Diagnostics, USA) is a recently developed chromogenic test for the diagnosis of BV that detects increased vaginal fluid sialidase activity in $10 \mathrm{~min}$. It is the first robust point-ofcare test available for BV and has a sensitivity of $90 \%$ and specificity of $95 \%$ compared to Nugent or Amsel scoring [10].

In this paper we present findings from the first feasibility study of antenatal point-of-care testing and treatment of CT, NG, TV and BV using a combination of GeneXpert CT/NG, TV and the BVBlue Test, provided in a routine antenatal clinic setting. This research was conducted in preparation for a large-scale field trial to evaluate this same strategy to improve pregnancy outcomes in PNG and other high-burden, LMIC settings.

\section{Methods \\ Study design and procedures}

A descriptive feasibility study was undertaken at a government-run urban antenatal clinic in Alotau, Milne Bay Province, PNG, between August and December 2014. All nursing staff at the clinic (Nursing Officers, Midwives and Community Health Workers) were trained in all aspects of the study, including the pointof-care testing.

Women aged 18 years or over and attending their first antenatal visit were invited to participate. Following written informed consent, women were enrolled consecutively and assigned a unique alphanumeric study identity (ID) number. Nursing staff conducted a short face-to-face interview with each participant, during which socio-demographic and sexual behaviour data were collected.

Women received routine antenatal and providerinitiated HIV and syphilis screening, and haemoglobin estimation. HIV testing was conducted using HIV rapid tests at point-of-care (Alere Determine HIV-1/2; followed by confirmatory Chembio Stat-Pak HIV-1/2), as per national guidelines. Syphilis testing used a rapid point-of-care screening test (SD Bioline anti-TP 3.0, Alere, Germany) followed by a confirmatory laboratorybased rapid plasma reagin (RPR) test. Women with a positive anti-TP plus a reactive RPR (any titre) test were considered to have active syphilis.

Following instructions from the nursing staff, participants provided two self-collected, mid-cavity, vaginal swabs for on-site testing: CT/NG and TV using the GeneXpert platform; and BV using the BVBlue rapid test assay. Testing was conducted by nursing staff supported by the in-country investigator team. Women diagnosed with any of these four infections were offered same-day 
antibiotic treatment in accordance with PNG STI management guidelines; risk-reduction counselling, and contact tracing was provided as necessary.

\section{Data management and statistical methods}

Study-specific case record forms (CRFs) were used to record socio-demographic, sexual behaviour, obstetric and clinical information. STI test results were automatically recorded in the GeneXpert laptop database by participant study ID number and were similarly recorded in a paper-based laboratory test log. CRF data were entered by study ID into a study-specific database without personal identifiers. Each day, all CRFs and test result log entries were reviewed for completion and consistency by the lead investigators. Clinical and STI test result data were entered into a study-specific Excel database and all electronic database entries validated against participant study folders for accuracy.

Data were summarised as frequencies and percentages. Fisher's exact test was used to compare statistical differences in outcomes of interest between younger and older women (e.g. STI prevalences). Data were analysed using Stata IC v14.0 (StataCorp LP, Texas, USA). Due to the modest sample size, it was not possible to conduct multivariate analysis to examine independent risk factors.

\section{Results}

Clinic staff were able to conduct point-of-care testing with minimal supervision after one day of intensive training. Interest in the study was high with nearly women who attended antenatal clinic requesting to participate. Due to a clinic schedule that allocates one weekday to new antenatal attendances (resulting in $20-25$ new visits on a single day); the availability of limited testing facilities (one single, four-module GeneXpert machine) and the requirement to provide same-day test results, we were unfortunately unable to enrol all those who asked to join. As such, 125 of the 222 (56\%) who attended the clinic were enrolled. Given these limitations women were enrolled as they presented to clinic, i.e. on a first come, first served basis and were not selected in any way by clinic staff or the research team. The integration of study procedures into routine antenatal clinic activities resulted in an average of two hours additional waiting time per woman.

Among the 125 women recruited, the median age was 24 years; around $75 \%(81 / 107)$ of women attended in the first or second trimester of pregnancy; and almost half were primigravid $(59 / 125 ; 47 \%$; Table 1$)$.

Province of birth was considered significant so as to identify what proportion of women had been born in the study province (Milne Bay) and what proportion were not, in order to look at differences in STI prevalence among these groups. 'Other province' includes all other provinces in PNG (there are 22 in total). Due to small numbers for each individual province we aggregated these and present this as 'Other province' rather than individual provinces.

Six women $(6 / 125 ; 5 \%)$ had a previous history of miscarriage, stillbirth or neonatal death. Based on point-of-care test results, more than half of women $(67 / 125 ; 53.6 \%)$ had any of CT, NG, TV or BV, among whom $71.6 \%$ (48/67) were asymptomatic. Overall, the prevalence of CT was $20.0 \%$; NG, $11.2 \%$; TV, $37.6 \%$; and BV, $17.6 \%$. The prevalence of HIV was $1.6 \%$, and $4.0 \%$ of the women had active syphilis. Women aged 24 years or under were significantly more likely to have any of CT, NG, TV or BV, compared with older women (63.6 \% vs. $42.4 \%$; odds ratio [OR] 2.38, $95 \%$ confidence interval $[\mathrm{CI}]: 1.09,5.21)$. Younger women with genital symptoms were six times more likely to have an infection compared with older women who had genital symptoms ( $25.8 \%$ vs. $5.1 \%$; OR 6.48, 95 \% CI: 1.70, 36.0). There were no significant differences between younger and older women in sexual behavioural characteristics, past history of an STI, or current genital symptoms. Among the 67 women who had one or more of CT, NG, TV or BV, there were no significant differences in sexual behavioural characteristics between younger and older women.

Among the 34 women who reported genital symptoms at enrolment, around half $(19 / 34 ; 55.9 \%)$ had any of CT, NG, TV or BV. Among the six women who reported a previous adverse pregnancy outcome, four had a history of stillbirth, of whom two had TV, and one had CT, TV and BV in the current study. All women received their test results the same day. Of those with any STI or genital infection, $83.6 \%(56 / 67)$ received same-day treatment.

The remaining $12.4 \%(11 / 67)$ had to leave prior to receiving treatment due to family commitments or the need to travel significant distances back to their homes by foot or by bus. All women had received treatment within one week. Partners in attendance at the clinic were treated the same day. All women and their partners diagnosed with an infection received treatment in accordance with PNG national guidelines [11]. Given the resource limitations of this study, test of cure follow-up was not possible. It will however be a part of future large scale antenatal trials.

\section{Discussion}

Antenatal point-of-care STI testing and treatment using a combination of novel, newly-available diagnostic tests proved feasible when integrated as part of routine antenatal care in this setting. This is the first study in any setting to evaluate the feasibility of this approach. The 
Table 1 Socio-demographic characteristics, sexual behaviour, obstetric history, genital symptoms and point-of-care test results

\begin{tabular}{|c|c|c|c|c|c|}
\hline \multirow{3}{*}{ Socio-demographic characteristics } & & \multirow{2}{*}{$\begin{array}{l}\text { Total } \\
N(\%)\end{array}$} & \multirow{2}{*}{$\begin{array}{l}\leq 24 \text { years } \\
N=66(\%)\end{array}$} & \multirow{2}{*}{$\begin{array}{l}\geq 25 \text { years } \\
N=59(\%)\end{array}$} & \multirow[t]{2}{*}{$p$ value } \\
\hline & & & & & \\
\hline & & & & & \\
\hline \multicolumn{2}{|l|}{ Age, median (IQR:18-41years) } & 24 years & 21 years & 30 years & \\
\hline \multirow[t]{2}{*}{ Province of birth } & Milne Bay Province & $100(80.0)$ & $54(81.8)$ & $46(78.0)$ & \\
\hline & Other province & $25(20.0)$ & $12(18.2)$ & $13(22.0)$ & \\
\hline \multirow[t]{2}{*}{ Marital Status } & Married & $108(86)$ & $51(77.3)$ & $57(96.6)$ & \\
\hline & Single & $17(13.6)$ & $15(22.7)$ & $2(3.4)$ & $p=0.002$ \\
\hline \multirow[t]{4}{*}{ Level of education $(N=124)$} & Never attended school & $4(3.2)$ & $0(0)$ & $4(6.7)$ & $p=0.032$ \\
\hline & Only primary school & $39(31.2)$ & $18(27.3)$ & $21(35.6)$ & \\
\hline & Secondary school & $81(65.3)$ & $47(71.2)$ & $34(57.6)$ & \\
\hline & Tertiary education & $24(19.2)$ & $9(13.6)$ & $15(25.4)$ & \\
\hline \multicolumn{6}{|l|}{ Obstetric history } \\
\hline \multirow[t]{3}{*}{ Parity $(N=124)$} & Primigravid & $59(47.6)$ & $48(72.8)$ & $11(18.6)$ & \\
\hline & Para 1-3 & $46(37.0)$ & $17(25.8)$ & $29(49.2)$ & $p<0.0001$ \\
\hline & Para 4 or more & $19(15.3)$ & $0(0.0)$ & $19(32.2)$ & $p=0.008$ \\
\hline \multicolumn{2}{|l|}{$\begin{array}{l}\text { Previous miscarriage/stillbirth/perinatal/ } \\
\text { neonatal death }(N=125)\end{array}$} & $6(4.8)$ & $3(4.5)$ & $3(5.1)$ & \\
\hline \multirow{4}{*}{$\begin{array}{l}\text { Estimated gestational age at enrolment } \\
\text { based on LMP }(N=107)\end{array}$} & 12 weeks or less & $13(11.2)$ & $5(7.6)$ & $8(13.6)$ & \\
\hline & 13-26 weeks & $68(63.5)$ & $38(57.6)$ & $30(50.1)$ & \\
\hline & $27-36$ weeks & $24(22.4)$ & $9(13.7)$ & $15(25.4)$ & \\
\hline & More than 36 weeks & $2(1.9)$ & $1(1.5)$ & $1(1.7)$ & \\
\hline \multicolumn{6}{|l|}{ Sexual behaviour } \\
\hline \multicolumn{2}{|l|}{ Age at sexual debut, median (IQR: 13-31years) } & 19 years & 18 years & 20 years & \\
\hline \multirow[t]{4}{*}{ Lifetime number of sexual partners $(N=119)$} & 1 partner & $37(31.1)$ & $20(30.3)$ & $17(28.8)$ & \\
\hline & 2-3 partners & $51(42.9)$ & $29(43.9)$ & $22(37.2)$ & \\
\hline & 4-6 partners & $21(17.6)$ & $11(16.6)$ & $10(16.9)$ & \\
\hline & 7 or more partners & $10(8.4)$ & $4(6.1)$ & $6(10.2)$ & \\
\hline \multirow[t]{3}{*}{ Sexual partners in previous month $(N=124)$} & 0 & $31(25.0)$ & $18(27.3)$ & $13(22.0)$ & \\
\hline & 1 & $80(64.5)$ & $41(62.1)$ & $39(66.1)$ & \\
\hline & 2 or more partners & $13(10.5)$ & $6(9.1)$ & $7(11.9)$ & \\
\hline \multirow{4}{*}{$\begin{array}{l}\text { Number of times had vaginal sex in the } \\
\text { last month }(N=122)\end{array}$} & 0 & $36(29.5)$ & $22(33.3)$ & $14(23.7)$ & \\
\hline & $1-2$ times & $28(23.0)$ & $11(16.7)$ & $17(28.8)$ & \\
\hline & 3 times or more & $32(26.2)$ & $17(25.6)$ & $15(25.4)$ & \\
\hline & More than once/unknown & $26(21.3)$ & $15(22.7)$ & $11(18.6)$ & \\
\hline \multirow[t]{2}{*}{ Condom use last sex $(N=124)$} & Yes & $29(23.4)$ & $12(18.2)$ & $17(28.8)$ & \\
\hline & No & $95(76.6)$ & $54(81.8)$ & $41(69.5)$ & \\
\hline \multicolumn{2}{|l|}{ Ever had sex for gifts/favours/money } & $31(24.8)$ & $16(24.2)$ & $15(25.4)$ & \\
\hline \multicolumn{6}{|l|}{ Reported genital symptoms at enrolment } \\
\hline \multicolumn{2}{|l|}{ Previous STI/genital infection } & $28(22.4)$ & $15(22.8)$ & $13(22.0)$ & \\
\hline \multirow[t]{5}{*}{ Current genital symptoms } & Any symptom & $34(27.2)$ & $20(30.3)$ & $14(23.7)$ & \\
\hline & Vaginal discharge ${ }^{a}$ & $24(19.2)$ & $16(24.2)$ & $8(13.6)$ & \\
\hline & Genital sores & $1(0.8)$ & $1(1.5)$ & $0(0.0)$ & \\
\hline & Abdominal pain ${ }^{\mathrm{a}}$ & $8(6.4)$ & $4(6.1)$ & $4(6.8)$ & \\
\hline & Itching $^{\mathrm{a}}$ & $19(15.2)$ & $10(15.1)$ & $9(15.3)$ & \\
\hline
\end{tabular}


Table 1 Socio-demographic characteristics, sexual behaviour, obstetric history, genital symptoms and point-of-care test results (Continued)

Other symptoms ${ }^{b}$

STI test results

\begin{tabular}{|c|c|c|c|c|}
\hline Any STI & $67(53.6)$ & $42(63.6)$ & $25(42.4)$ & $p=0.017$ \\
\hline $1 \mathrm{STI}$ & $39(31.2)$ & $22(33.3)$ & $17(28.8)$ & \\
\hline 2 or more STIs & $28(22.4)$ & $20(30.3)$ & $8(13.6)$ & $p=0.025$ \\
\hline HIV & $2(1.6)$ & $2(3.0)$ & $0(0.0)$ & \\
\hline Active syphilis ${ }^{c}$ & $5(4.0)$ & $3(4.5)$ & $2(3,4)$ & \\
\hline C. trachomatis $(\mathrm{CT})$ & $25(20)$ & $18(27.3)$ & $7(11.9)$ & $p=0.032$ \\
\hline N. gonorrhoeae (NG) & $14(11)$ & $12(18.2)$ & $2(3.4)$ & $p=0.009$ \\
\hline T. vaginalis (TV) & $47(37.6)$ & $31(47.0)$ & $16(27.1)$ & $p=0.022$ \\
\hline Bacterial vaginosis (BV) & $22(17.6)$ & $11(16.7)$ & $11(18.7)$ & \\
\hline Genital symptoms and 1 or more STI detected & $19(15.2)$ & $16(24.3)$ & $3(5.1)$ & $p=0.002$ \\
\hline Genital symptoms but no STI detected & $15(12.0)$ & $4(6.1)$ & 11 (18.6) & $p=0.031$ \\
\hline
\end{tabular}

${ }^{\mathrm{a}} 14$ women had multiple symptoms/ ${ }^{\mathrm{b}}$ discharge, pus, "wetness"/Canti-TP+ and RPR+, all titres

high interest shown in study participation and $100 \%$ completion of study procedures among those enrolled suggest the intervention has a high level of acceptability, but needs to be formally established. An important limitation was our inability to meet antenatal testing demand: we were only able to recruit around $56 \%$ of those who attended yet nearly all women expressed an interest in joining the study. Future intervention studies and demonstration projects should consider setting up more than one four-module GeneXpert machine per clinic in situations of high workload.

High prevalences of STIs and genital infections were observed among pregnant women in this setting, particularly those aged 24 years or under. These findings are consistent with our earlier research among antenatal women in PNG $[12,13]$. A minority of women (34/125; $27.2 \%$ ) in the current study reported genital symptoms at enrolment, of whom around half had CT, NG, TV or $\mathrm{BV}$; whilst more than $70 \%$ of women with one or more of these curable infections were asymptomatic. These findings suggest that STI syndromic management, based on clinical presentation alone, is a poor strategy for the detection and treatment of STIs among pregnant women in this setting and underline the need for robust, easyto-use, timely, and accurate point-of-care tests.

In this pilot study, the cost-effectiveness of this new and broad based POC testing approach was not examined in detail. A cluster randomised trial of antenatal point-of-care testing and treatment to improve pregnancy outcomes is due to start in PNG in 2016 [13]. This trial will establish the efficacy, acceptability, costeffectiveness and health system requirements of the proposed test and treat strategy, and will inform future international policy and practice on the diagnosis and management of STIs in pregnancy.

\section{Conclusions}

With increased Genexpert device availability this integrated point of care testing strategy has the potential to change the way STI's in pregnancy are managed and reduce the number of associated adverse health outcomes for antenatal women in Papua New Guinea.

\begin{abstract}
Abbreviations
BV, Bacterial vaginosis; $C T$, Chlamydia trachomatis; CRF, Case record form; HIV, Human Immunodeficiency Virus; LMIC, Low middle income country; NAAT, Nucleic acid amplification test; NHMRC, National Health and Medical Research Council; NG, Neisseria gonorrhoeae; PNG, Papua New Guinea; POC, Point of care; RPR, Rapid plasma reagin; STI, Sexually transmitted infection; TV, Trichomonas vaginalis; WHO, World Health Organisation
\end{abstract}

\section{Acknowledgments}

We are extremely grateful to all the women who took part in this research and to their families and communities for supporting this project. We would especially like to thank the clinic staff involved in this work, without whom this study would not have been possible: Ester Gibson, Rose Joshua, Jane Raurela, Hadassha Dugutara, Leanne Raurela, Janet Adrian, Hilda Henry, Noreen Tabua, Faith Malesa, Catherine Wallace, Amos Giarua, Angela Kamnanaya and Jenny Poela. We also acknowledge the invaluable support and guidance provided by the Milne Bay Provincial Health Authority and Alotau General Hospital, in particular: Billy Naidi, Dr Jacob Morewaya and Dr Noel Yaubihi.

Funding

This study was funded under a Program Grant from the National Health and Medical Research Council, Australia.

Availability of data and materials

All relevant data from this study has been published in this manuscript. Identifying or confidential patient data will not be shared.

\section{Authors' contributions}

$\mathrm{SB}, \mathrm{AV}, \mathrm{LV}$ and PT were the primary investigator team and provided point-ofcare training and supervision of this research over the duration of the study. GK, WP, GM provided provincial and national level assistance in the formation of this study and liaison with key in-country stakeholders. SL, CM, SG, SR, $B D, L C, R G, C H$ and JK provided guidance with the study design and related technical issues. $\mathrm{BL}$ is the senior clinic manager and provided direction to clinic staff, supporting the integration of POC testing into the clinic workflow. 
DW and ST advised on laboratory techniques. HW supported the statistical analysis of data. All authors reviewed and approved the final manuscript.

\section{Competing interests}

The authors declare that they have no competing interests.

\section{Consent for publication}

Not applicable.

\section{Ethical approval and consent to participate}

Ethical approval was provided by the Medical Research Advisory Committee of the PNG National Department of Health (MRAC\#14.28), the Institutional Review Board of the PNG Institute of Medical Research (IRB\#1406), the Milne Bay Provincial Research Ethics Committee in PNG (letter of approval 30 July 2014); and by the Human Research Ethics Committee of UNSW Australia (HREC UNSW \#HC14118). Written informed consent (signature or witnessed thumbprint) was obtained from all participants prior to enrolment.

\section{Author details}

${ }^{1}$ The Kirby Institute, University of New South Wales, Sydney, Australia. ${ }^{2}$ Papua New Guinea Institute of Medical Research, Goroka, Papua New Guinea. ${ }^{3}$ Department of Obstetrics \& Gynaecology, Alotau General Hospital, Milne Bay Province, Alotau, Papua New Guinea. ${ }^{4}$ Alotau Urban Clinic, Alotau, Milne Bay Province, Papua New Guinea. ${ }^{5}$ University of Technology, Sydney, Australia. ${ }^{6}$ The Burnet Institute, Melbourne, Australia. ${ }^{7}$ Melbourne School of Population and Global Health, University of Melbourne, Melbourne, VIC, Australia. ${ }^{8}$ Department of Microbiology and Infectious Diseases, The Royal Women's Hospital, Parkville, VIC, Australia. ${ }^{9}$ Department of Obstetrics and Gynaecology, University of Melbourne, Murdoch Children's Research Institute, Parkville, VIC, Australia. ${ }^{10}$ University of Queensland, Herston, QLD, Australia. ${ }^{11}$ Department of Medicine, University of Melbourne, Melbourne, Australia.

${ }^{12}$ Department of Obstetrics \& Gynaecology, School of Medicine and Health Sciences, University of Papua New Guinea, National Capital District, Papua New Guinea. ${ }^{13}$ Sydney Sexual Health Centre, Sydney, Australia.

Received: 18 December 2015 Accepted: 17 May 2016

Published online: 06 June 2016

\section{References}

1. Mullick S, Watson-Jones D, Beksinska M, Mabey D. Sexually transmitted infections in pregnancy: prevalence, impact on pregnancy outcomes, and approach to treatment in developing countries. Sex Transm Infect. 2005;81: 294-302.

2. UNICEF, WHO, Bank W, UnitedNations. Levels \& Trends in Child Mortality: Estimates Developed by the UN Inter-agency Group for Child Mortality Estimation. New York: UNICEF; 2012

3. NDoH, National Health Information Systems. Sector Performance Annual Review 2013. Papua New Guinea: National Department of Health; 2014.

4. UNFPA, ICM, WHO. The State of the Worlds Midwifery. A universal pathway. A woman's right to health. New York: United Nations; 2014.

5. Vallely A: Sexually transmitted infections in Papua New Guinea: Achievements, challenges and the way forward. In: 2014 Australasian Tropical Health Conference. Cairns; 2014

6. WHO. Guidelines for the Management of Sexually Transmitted Infections, vol. WHO/RHR/01.10. Geneva: World Health Organization; 2001.

7. WHO. Global strategy for the prevention and control of sexually transmitted infections: 2006-2015: breaking the chain of transmission. Geneva: World Health Organization; 2007.

8. Tabrizi SN, Unemo M, Golparian D, Twin J, Limnios AE, Lahra M, Guy R, Investigators T. Analytical evaluation of GeneXpert CT/NG, the first genetic point-of-care assay for simultaneous detection of Neisseria gonorrhoeae and Chlamydia trachomatis. J Clin Microbiol. 2013;51(6):1945-7.

9. WHO Guidelines Approved by the Guidelines Review Committee. In: Policy Statement: Automated Real-Time Nucleic Acid Amplification Technology for Rapid and Simultaneous Detection of Tuberculosis and Rifampicin Resistance: Xpert MTB/RIF System. edn. Geneva: World Health Organization. Copyright (c) World Health Organization 2011.; 2011.

10. Bradshaw CS, Morton AN, Garland SM, Horvath LB, Kuzevska I, Fairley CK. Evaluation of a point-of-care test, BVBlue, and clinical and laboratory criteria for diagnosis of bacterial vaginosis. J Clin Microbiol. 2005;43(3):1304-8.
11. Mola GDL: Manual of Standard Managements in Obstetrics and Gynaecology for Doctors, HEOs and Nurses in Papua New Guinea, Sixth Edition edn; 2010

12. Wangnapi RA, Soso S, Unger HW, Sawera C, Ome M, Umbers AJ, Ndrewei N, Siba P, Li Wai Suen CS, Vallely A et al. Prevalence and risk factors for Chlamydia trachomatis, Neisseria gonorrhoeae and Trichomonas vaginalis infection in pregnant women in Papua New Guinea. Sex Transm Infect. 2015;91(3):194-200.

13. Vallely AJ: Point-of-care testing and treatment of sexually transmitted infections to improve pregnancy outcomes in high-burden settings..In: World STI \& HIV Congress 2015. Brisbane, Australia; 2015

\section{Submit your next manuscript to BioMed Central and we will help you at every step:}

- We accept pre-submission inquiries

- Our selector tool helps you to find the most relevant journal

- We provide round the clock customer support

- Convenient online submission

- Thorough peer review

- Inclusion in PubMed and all major indexing services

- Maximum visibility for your research

Submit your manuscript at www.biomedcentral.com/submit 